\title{
From Cultured Rodent Neurons to Human Brain Tissue - Model Systems for Pharmacological and Translational Neuroscience
}

\author{
by \\ Joel Wellbourne-Wood and Jean-Yves Chatton \\ Department of Fundamental Neurosciences, University of Lausanne, \\ 1005 Lausanne, Switzerland
}

${ }^{*}$ Corresponding author:

Jean-Yves Chatton, PhD

Dept. of Fundamental Neurosciences

University of Lausanne

Rue Bugnon 9

Ch-1005 Lausanne, Switzerland

Tel. +41-21-692-5106 Fax: +41-21-692-5105

E-mail: jean-yves.chatton@unil.ch

Keywords: tissue culture; brain slices; primary cell culture; model systems; neurons; astrocytes 


\section{For Table of Contents Use Only}

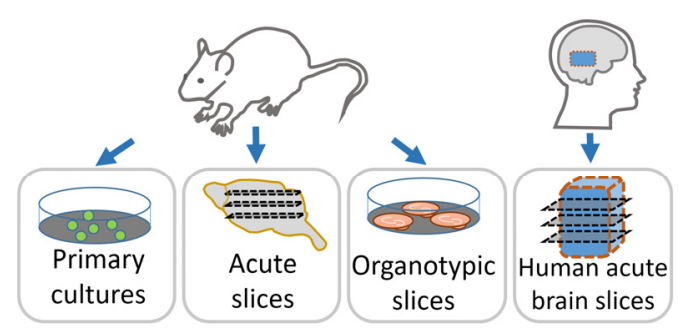

From Cultured Rodent Neurons to Human Brain Tissue - Model Systems for Pharmacological and Translational Neuroscience

Joel Wellbourne-Wood and Jean-Yves Chatton 


\section{Abstract}

To investigate the enormous complexity of the functional and pathological brain there are a number of possible experimental model systems to choose from. Depending on the research question choosing the appropriate model may not be a trivial task, and given the dynamic and intricate nature of an intact living brain several models might be needed to properly address certain questions. In this review we aim to provide an overview of neural cell and tissue culture, reflecting on historic methodological milestones and providing a brief overview of the state-of-the-art. We additionally present an example of an effective model system pipeline, composed of dissociated mouse cultures, organotypics, acute mouse brain slices, and acute human brain slices, in that order. The sequential use of these four model systems allows a balance and progression from experimental control to human applicability, and provides a meta-model that can help validate basic research findings in a translational setting. We then conclude with a few remarks regarding the necessity of an integrated approach when performing translational and neuropharmacological studies. 


\section{Introduction}

The adult human brain is composed of approximately 80 billion neurons and $40-50$ billion glial cells ${ }^{1}$, while overall brain size computed using a number of factors differs 100,000 fold across the mammalian class $^{2}$. It is the most energetically demanding organ in the body- despite the fact that it makes up around $2 \%$ of the body by weight, it demands nearly $20 \%$ of oxygen mobilized in the bloodstream, most of which enables the generation of action potentials, the reestablishment of pre-synaptic ion gradients, and the generation of post-synaptic ion fluxes ${ }^{3}$.

The brain is also one of the most dynamic organs over a developmental time course, and can undergo substantial rearrangement well into adulthood ${ }^{4}$. Precursor cells known as neuroblasts give way to neurons and glial cells that will compose both the central and peripheral nervous systems. Unlike glial cells, neurons typically do not divide, but rather develop a range of involved and patterned branches and arborizations known as dendrites and axons. These branches follow complex pathfinding programs coordinated by attractive and repulsive signaling molecules ${ }^{5}$, undergo pruning and refinement ${ }^{6}$, and generate complex networks, ranging from microscopic cellular arrangements such as cortical layers and columns, up to macroscopic and hemispheric divisions that govern encompassing processes such as the visual and motor cortices.

Taking into account the spatial and temporal intricacy of the nervous system, as a functional structure, it may be the most complex we have discovered so far in our universe. Additionally, current neuroscientific methodology for in-vivo research is limited by several factors, including the fragility and sensitivity of the brain to invasive procedures, relative optical opacity of the skull and brain tissue, permeability of the blood-brain-barrier, and more. While some methods are not affected by these limitations or have been designed around them (EEG, for example), other methods such as imaging or electrophysiology remain challenging. Due to the conceptual and practical complexities associated with studying the brain its systematic deconstruction, and the iteration of neuronal and cell-typemixed model systems, has expanded outwards in different directions, yielding a range of creative and varied models as a result. This complexity also applies to research into pathological circumstances, as in many cases of neurological disorder the etiology and path forward are not clear.

In this review we aim to provide a brief overview of the development of model systems used to study the normal and pathological functioning of the brain to date, and to propose an integrated model system approach aimed especially at researchers whose goal is to validate their findings from other mammals such as rodents, and to translate fundamental research into drug targets or potential therapies in humans. 


\section{An Overview of Neuronal Model Systems}

\section{The Rise of Neuronal Cell Culture}

The first ex-situ preparations of neurons designed for live growth outside of the body were the first such preparations of any multicellular eukaryote in history ${ }^{7}$. The system used, namely the hanging drop (figure 1a), was a preparatory protocol devised by Ross Harrison and first published on in the year 1907. This was a landmark study in which small resected pieces of embryonic frog tissue, which at the time were "known to give rise to nerve fibers" were placed in an indentation on a glass coverslip. A drop of lymph was then applied to the tissue, which clots and holds the tissue in place, also providing a satisfactory nutritional and electrolytic composition. Under these conditions Harrison was able to keep the tissue alive for between one and four weeks, during which he characterized the differential growth patterns of nerves derived from distinct anatomical regions of the embryo. Only later was the hanging drop strategy applied to non-neuronal cell biology studies ${ }^{8}$. This was one of the first systems devised that demonstrated not only that single cells and a variety of tissues derived from multicellular eukaryotes were able to be removed from the body without cell death, but also that they could be cultured and propagated. Later, in an effort to simplify and improve cell culture techniques, a range of culture flasks were designed. The precursor to the modern culture dishes were the Carrel flasks, intended to give more experimental control to the researcher (figure 1b). This included easier access to the cultures, more medium bathing the tissue, greater surface area, and improved longevity ${ }^{9}$. Carrel flasks have since been improved upon, resulting in modern culture flasks used today. Improvements include being manufactured out of plastic instead of glass, marked with volumetric indicators, and standardized in size. They are also no longer equipped with cotton stoppers, but rather with screw caps, sometimes including semi-permeable membranes to allow for gas exchange.

Further developments meant that lymph as a medium was replaced by predefined salt solutions mixed with agar and chicken stock ${ }^{10}$, a solution based on the ones composed by Ringer and Buxton in 1885 to investigate acutely resected frog hearts ${ }^{11}$. The additional agar and factors in the chicken stock promoted longevity of cultures and allowed more defined media to become standardized, improving reproducibility and experimental control. In 1955 Harry Eagle published a paper on minimum essential medium (MEM), in which $\mathrm{pH}$ buffering agents were introduced ${ }^{12}$. Today a modified version of MEM known as Dulbecco's modified MEM (DMEM) is still widely used. Serum from bovine, horses, and specifically fetal calves is often supplemented to improve culture growth and longevity by providing growth factors, hormones, vitamins, trace elements, and more ${ }^{13}$. More recently efforts have been made to use serum-free culture media, replacing components of biological origin with known factors 14. Media that cater specifically to neuronal demands have also been designed, such as the B27 supplemented Neurobasal medium ${ }^{15}$, and the more recently developed BrainPhys medium, based on 
an improved formulation ${ }^{16}$. To mimic physiological conditions, cell culture media $\mathrm{pH}$ is typically maintained using a bicarbonate buffer, characterized by the balance between bicarbonate $\left(\mathrm{HCO}_{3}{ }^{-}\right)$, carbonic acid $\left(\mathrm{H}_{2} \mathrm{CO}_{3}\right)$, and $\mathrm{CO}_{2}$, requiring an incubator with controlled $\mathrm{CO}_{2}$ levels, generally $5 \%$. Another development was the Hibernate E medium, which maintains neural tissue viability for several days at ambient $\mathrm{CO}_{2}$, and up to several weeks while refrigerated, greatly facilitating storage and transport of neuronal cultures and tissues ${ }^{17}$. These innovations in cell culture viability, reproducibility, and practical implementation provided the foundation for modern neuronal culture.

a

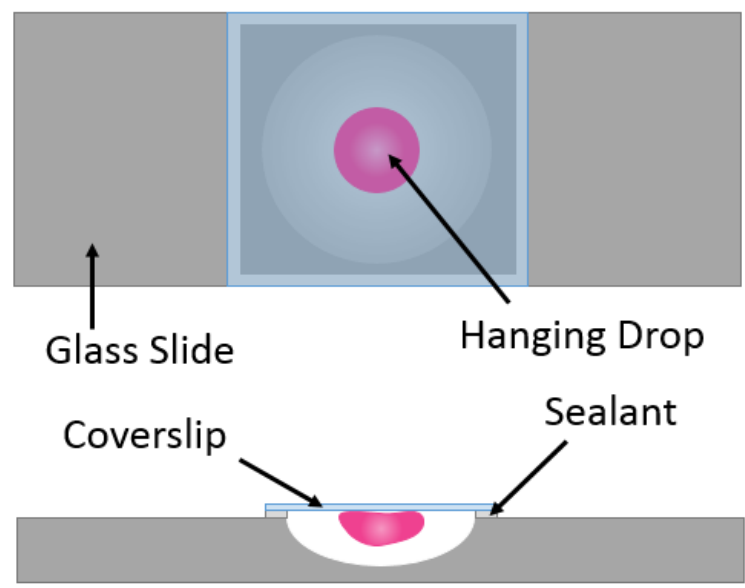

b

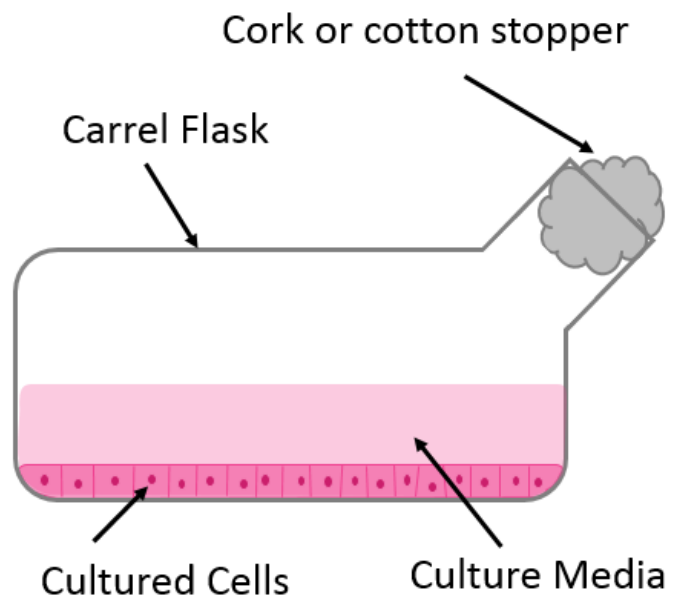

Figure 1. Scheme depicting historic cell culture milestones. (a) the hanging drop, composed of tissue contained in a clotted drop of lymph, situated in the groove of an indented glass slide, hanging from a coverslip attached to the slide by sealant such as grease, and (b) The Carrel Flask, containing cultured cells and growth media, with a canted neck designed for easier maintenance and accessibility, as well as a cork or cotton stopper to allow for gas exchange while still protecting against contamination.

\section{Directed Growth and Cellular Manipulation}

In order to generate the right model system, the manipulation of neural cell cultures intrinsic properties is often necessary. Through the use of transfections, infections, electroporation, and microinjections ${ }^{18}$, as well as de-novo mutations in transgenic mouse lines ${ }^{19}$, the introduction of genetic elements such as coding sequences, shRNA, and more can be controlled. This facilitates the generation of cell cultures that express channels, receptors, or any specific gene of interest, either as mutated forms or their wild-type counterparts, for functional binding, signaling, and electrophysiological studies. 
As the field of cellular neuroscience develops the ability to finely manipulate the external setting and microenvironment of cells also becomes increasingly desirable. This includes regulating the extracellular microenvironment and controlling cellular development and interactions via external cues, as well as growth conditions. Efforts to control cell-cell interactions and single cell dynamics led, for example, to the development of the micro-island culture method. While investigating neuroncardiomyocyte interactions and following the idea that "the incidence of detectable interaction would increase if the innervation field of a given neuron was concentrated," the researchers developed glass coating protocols that allowed cell adhesion only at small sites, spanning the range of only a few hundred micrometers, resulting in "islands" composed of single cells or groups of just a few cells ${ }^{20}$ (figure 2). As a model system the micro-island introduced a greater degree of reductionism to in-vitro work on the brain, allowing for a finer dissection of cellular and subcellular interactions. Micro-islands have been used to study a range of topics including but not limited to synapse maturation ${ }^{21,22}$, the role of connectivity on transmission ${ }^{23,24}$, single-cell epileptiform dynamics ${ }^{25}$, and heterogeneity of active release sites ${ }^{26,27}$.
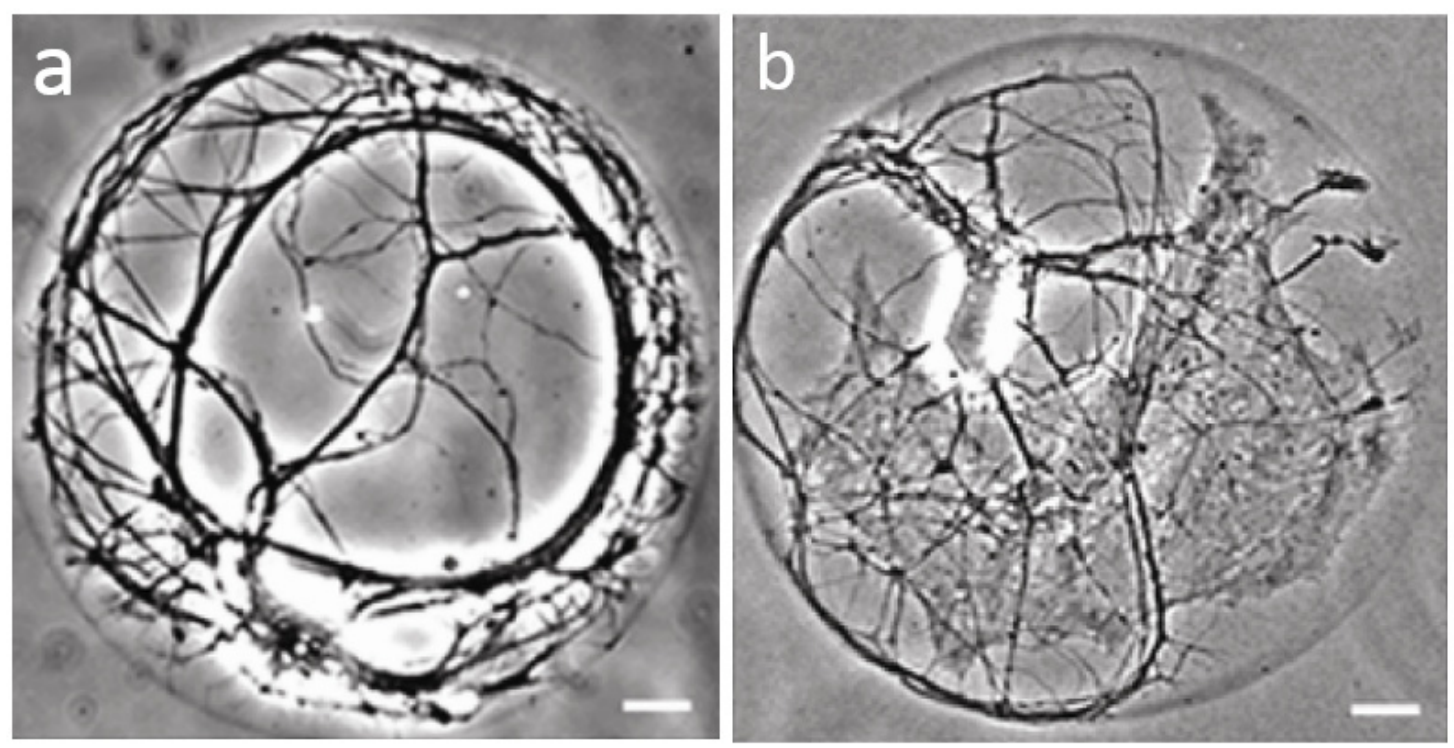

Figure 2. Example of micro-island neuronal cultures. Light microscopy images show (a) a single rat cortical neuron and (b) a single neuron with an accompanying astrocyte, growing on a droplet of permissive substrate (poly-D-Lysine and collagen) deposited on top of an agarose-coated glass coverslip. The neurites and cell processes remain confined to the disk of substrate as their growth is inhibited by the agarose coating of the coverslip. Scale bars, $10 \mu \mathrm{m}$. Other micro-island configurations such as two connected neurons are also possible. (Modifed with permission from F.D. Morgenthaler ${ }^{26}$, Univ. Lausanne).

The natural evolution of the controlled growth paradigm also led to the development of microfluidic devices, culture chambers that house compartments or channels of microscopic dimensions ${ }^{28}$. The introduction of new advances in microfabrication techniques and their ease of implementation meant 
that creative design solutions can now be implemented to overcome geometric limitations of conventional cell culture methods. This possibility allows microenvironmental control of cytoarchitecturally distinct regions of individual neurons such as dendrites and axons ${ }^{29}$, as well as cell assemblies, such as injured nerve fibers ${ }^{30}$ or neuromuscular units ${ }^{31}$, through the controlled delivery of microliters or nanoliters of compositionally distinct solutions to individual compartments. This allows the model system to mimic multiple environmental states, such as are found in different brain regions ${ }^{32}$, or in healthy vs diseased states ${ }^{33}$, making microfluidics a potentially powerful experimental system for pharmacological and translational studies. For example, a recent publication utilizing a socalled "on-a-chip" in-vitro corticostriatal neuronal network using microfluidic techniques demonstrated that the presynaptic cortical component of the network plays a larger role in Huntington's Disease (HD) pathology than previously thought ${ }^{34}$. The authors also remark on the benefits of their on-a-chip system, such as its suitability for high temporal and spatial resolution imaging, testing drugs for treatment of HD, and its applicability to other pathologies. It is now well established that glial-derived factors play a large role in neuronal development and maintenance ${ }^{35-40}$, which has led to methodological development of co-culture techniques ${ }^{41,42}$ designed to better mimic conditions found in the brain. Co-cultured glial cells and neurons are often grown on separate surfaces and only share the media they are submerged in (figure 3a), while other co-culture methods will have cells in physical contact (figure $\mathbf{3 b}$ ). Of note is that co-culture techniques are compatible with both micro-island cultures ${ }^{43,44}$ (figure $\mathbf{2 b}$ ) and microfluidic chambers ${ }^{45,46}$. Advantages of co-cultures can also be reaped for translational and pharmacological research. One such study reported on a human cell line co-culture model of Parkinson's Disease (PD) with a pharmacological profile demonstrating it was a "closer model of human brain tissue than conventional cultures," and, "Its use for screening of candidate neuroprotectants may increase the predictiveness of a test battery" ${ }^{\prime 4}$. Another study established a blood-brain- barrier model via co-culture techniques using human induced pluripotent stem cells (hIPSCs) and fetal neural stem cells, performing permeability testing with reference substances to demonstrate the relevance of the model for drug transport studies ${ }^{48}$. 
a

No Contact

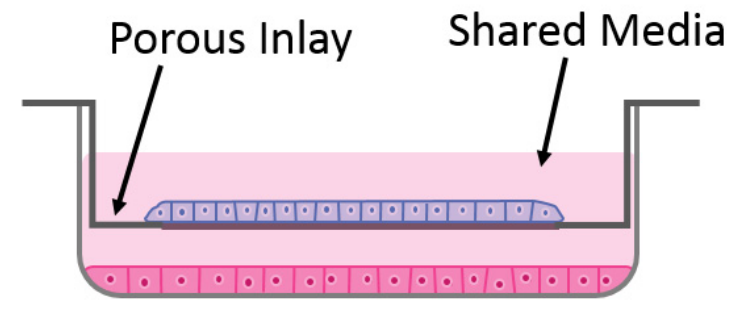

Conditioned Media Replacement

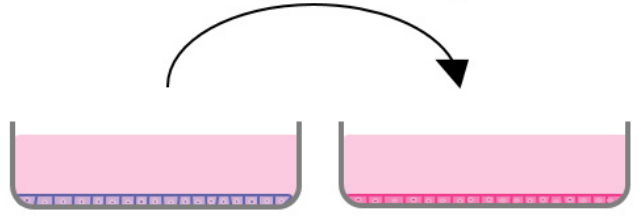

b Contact

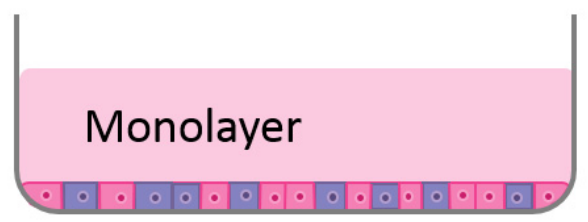

3-Dimensional

Figure 3. Scheme depicting co-culture variants including (a) methods without direct cell-cell contact that rely solely on soluble factors from shared media, either via porous inlays (top) or conditioned media replacement (where compositionally conditioned culture media is removed from one set of cells and used to culture another; bottom), and (b) methods that result in direct cell-cell contact, such as mixed monolayer cultures (top) and 3-dimensional mixed cultures. Neurons may be grown on a feeder layer of astrocytes, for example. (bottom).

The hIPSC discovery advanced the use of human derived cell lines considerably. The initial finding that the transduction of just four transcription factors into cultured adult and embryonic fibroblasts could induce pluripotency ${ }^{49}$ led to a surge of research into genetic lineage reprogramming technology and methodology, leading to a simultaneous report by two groups of human cell reprogramming ${ }^{50,51}$, and later direct lineage reprogramming of somatic cells into neural stem cells or neurons, removing labor intensive intermediate steps associated with pluripotent cell culture and maintenance ${ }^{52,53}$. It has also been demonstrated that urine derived cells can be directly reprogrammed into neurons ${ }^{54}$. Using less invasive somatic cell collection methods such as urine collection makes reprogramming neurons from a wider range of patients a viable strategy. The ability to culture neurons containing the genetic code of specific and living cohort subjects, as well as patients, may not only help fundamental and translational research, but may also play a larger role in personalized medicinal strategies going forward. 


\section{The Acute Brain Slice}

One of the most widespread model systems in neuroscience is the acute brain slice ${ }^{55-57}$. Living brain tissue cut into thin sheets between 100-400 $\mu \mathrm{m}$ in thickness can be kept alive in incubation chambers mimicking natural conditions in the brain and can be used over an experimental session lasting several hours. Typical protocols involve the acute preparation of brain slices, usually from juvenile animals unless the experimental question demands older ones, and their subsequent incubation in oxygenated, buffered salt solutions that mimic cerebrospinal fluid. Although ice-cold cutting solutions are the methodological norm, cutting at physiological temperatures has also been established ${ }^{58}$. Incubation of the slices is commonly performed at room temperature, while recordings are often performed closer to physiological temperatures. It is also important to consider cutting orientation, since both the functional connectivity between cells, or between cell assemblies via fibers, should be preserved as much as possible. This may require protocol optimization and practice, as well as preexisting knowledge about the circuitry and anatomy.

Acute slices can also be prepared from transgenic animals such as those designed to act as models of specific pathologies, or that have mutated, knocked-in, or knocked-out genes. Behavioral data from specific mice can also be correlated with data obtained from the slices of those animals, such as functional or morphological data. They are also often prepared from animals genetically engineered for optogenetic applications, which allows for ex-vivo dissection of behavior and condition related circuitry 59,60 . Brain Slices can also be acutely loaded with fluorescent ion indicators or other fluorescent dyes.

The power of the acute brain slice model lies in its functional connectivity and relative homology to the intact brain. This enables research into specific systems, something dissociated cell cultures lack. The model has provided insight into thalamocortical pathways ${ }^{61,62}$, olfactory network activity ${ }^{63-65}$, and hippocampal circuitry ${ }^{66-68}$, just to name a few of many research avenues that employ acute slices. In terms of the model's advantage over intact, living animals, acute slices permit a large degree of experimental control. The ability to provide drugs without a blood brain barrier, to visualize specific cells and structures, and to perform more involved electrophysiological or pharmacological studies make it a versatile system. Answering specific research questions that require the identification of cell types, the control of the extracellular microenvironment, and a wider range of measurements is therefore greatly facilitated, allowing for fine quantitative studies of mechanistic and conditional processes ${ }^{69}$. 


\section{Brain Organoids and Tissue Culture}

Although acute brain slices constitute a powerful neuroscience model system they suffer from a relatively low throughput rate. Every experimental session requires more preparation, and tissue remains viable for a few hours, or up to three days using advanced environmental control methods ${ }^{70}$. Acute slices are relatively short-lasting, so in order to achieve a higher throughput and reduce the number of animals used, all while retaining connectivity and 3-dimensionality, one may turn to organoid and tissue cultures methods. Organoid cultures are 3-dimensional cultures that are typically grown using either human or animal IPSCs or embryonic stem cells (ESCS) differentiated into neural tissue. They provide models that not only successfully recapitulate early stages of human and animal brain development at a genetic and single-cell level ${ }^{71}$, but also provide some degree of 3D cytoarchitectural homology with real brains, and have already been used to investigate the development of a number of pathologies ${ }^{72}$. Like normal cell culture methods, organoid cultures can be maintained in culture for much longer than acute slices.

a

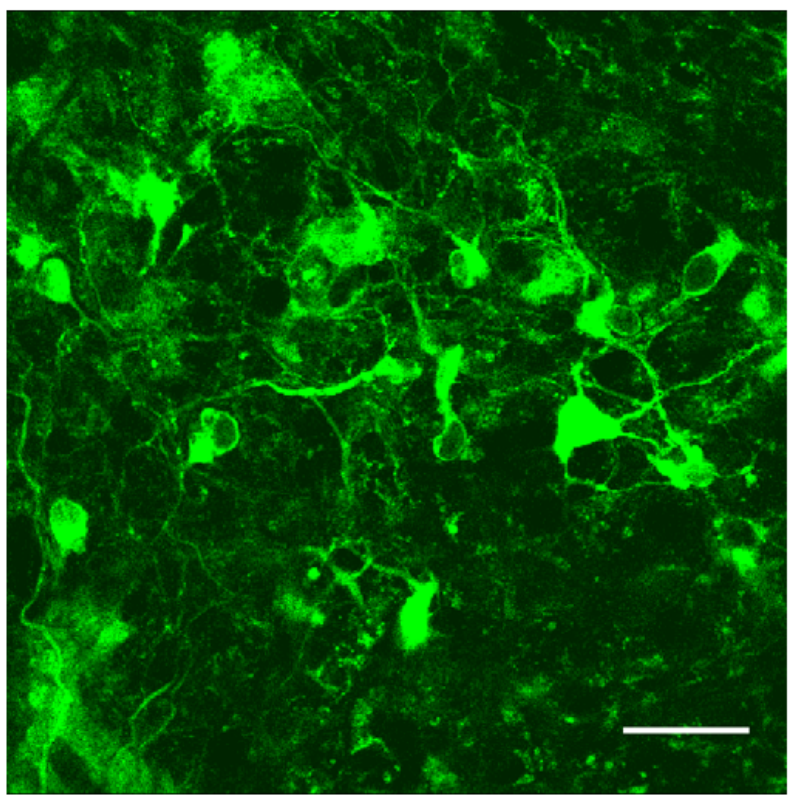

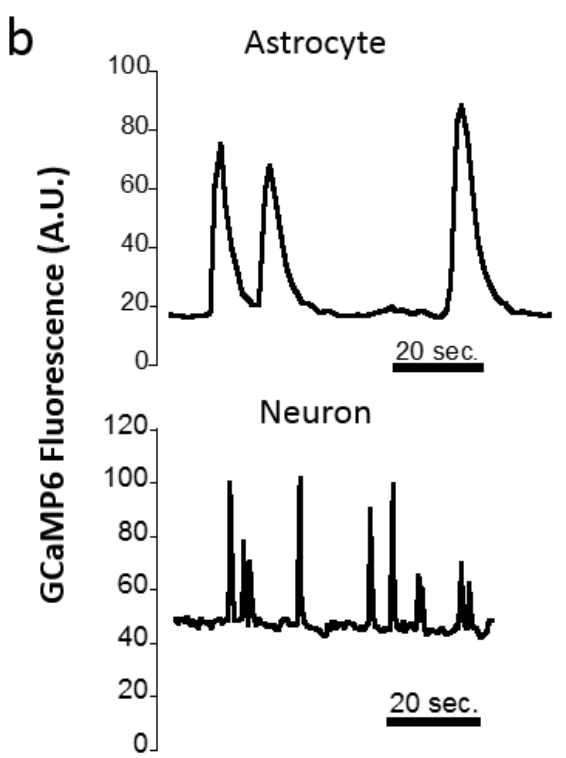

Figure 4. Example of organotypic slice infected with GCaMP6, a green fluorescent genetically encoded calcium indicator, including (a) a confocal image of a hippocampal organotypic slice $\left(\lambda_{\text {ex }} 488 \mathrm{~nm}\right)$, scale bar $=20 \mu \mathrm{m}$, and (b) typical fluorescence traces from an astrocyte (top) and a neuron (bottom) during real-time calcium imaging.

The organotypic slice culture has properties that lie somewhere in-between dissociated cell cultures and acute brain slices. They are cultured resections of tissue that generally retain typical morphological structure associated with the original organ, even after prolonged culture. An example would be the 
hippocampus, which can be resected, sliced into thin sections, and cultured. As a model system organotypics became more widespread after the advent of porous membranes on which the tissue could be cultured, which reduced the difficulty and increased the reproducibility of the model ${ }^{73}$. The first reference to organotypics in neuroscience was a study focused on cerebellar tissue ${ }^{74}$, and since then they have been produced from various regions of the brain including but not limited to the cortex ${ }^{75}$, substantia nigra ${ }^{76}$, striatum ${ }^{77}$, olfactory neuroepithelia ${ }^{78}$, and the hippocampus ${ }^{79}$. Since a range of brain areas can be investigated using organotypic cultures, it stands to reason that they might be used to model a range of neuropathologies. For example in terms of Alzheimer's Disease (AD), organotypics have been used to investigate signaling and toxicity ${ }^{80,81}$, oxidative stress ${ }^{82}$, tau phosphorylation ${ }^{83}$, and variables affecting $A D$ development in-vitro such as age ${ }^{84}$ and growth factors ${ }^{85}$. Organotypics have also been used to model PD ${ }^{86-88}$, ischemic injury ${ }^{89,90}$, and other pathological conditions ${ }^{91,92}$. Since organotypics can be cultured for significant periods of time, they are also useful for long-term observation and can also be genetically modified with relative ease, for example using genetically encoded sensors (figure 4).

\section{A Model System Pipeline for Translational Neuropharmacology}

\section{Necessity of an Integrated Approach}

A general point of concern regarding translational neuroscience is the broadness of applicability of laboratory findings. Discoveries in cell culture models may not apply to the acute slice, and discoveries in the acute slice may not apply to intact animals. Finally, the degree of similarity between animals and humans needs to be evaluated in regards to specific questions when performing translational research.

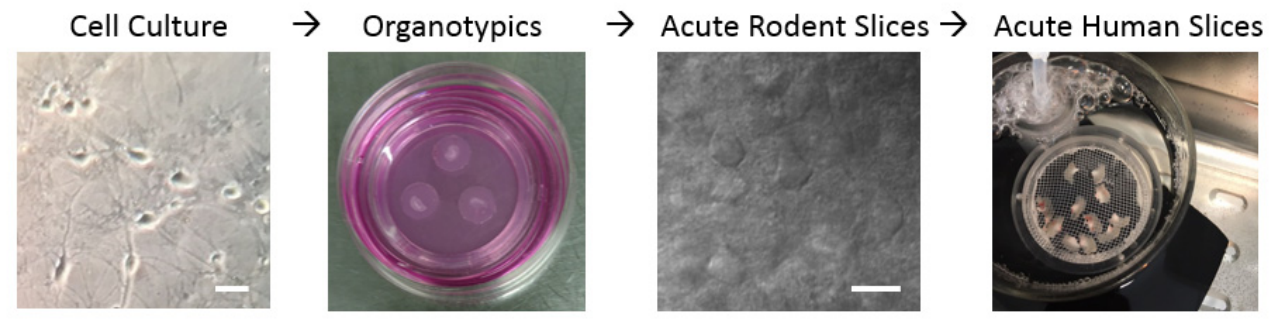

\section{Translational Relevance}

\section{Experimental Control}

Figure 5. A progressive neuronal model system pipeline, composed of cell culture (scale bar $=20 \mu \mathrm{m}$ ), organotypic slices, acute rodent slices (scale bar $=20 \mu \mathrm{m}$ ), and acute human slices. As a study transitions from one model system to the next, experimental control is decreased, but translational relevance is gained. 
As a result, findings need to be carefully evaluated using a range of model systems, especially when they pertain to potential drug targets and therapies. As an example of the degree of congruency between laboratory findings and biomedical advances, the average percentage of translated cancer studies aiming to find therapies for humans based on animal results that pass phase 1 trails is just $8 \%$

93. One way to increase the likelihood of success in specific cases and to accelerate discovery of incongruence between different models and organisms is to design an integrated model system pipeline, where several model systems` strengths are used to support weaknesses of others within a body of research.The models we propose integrating are the dissociated neuronal cell culture, rodent organotypics, acute rodent brain slices, and acute human brain slices (Figure 5). Depending on the type of research being conducted one may develop another effective model system pipeline. As our laboratory is currently focused on metabolic and neuropharmacological research that may also be relevant to human pathology, we use a sequence of model systems that provide good experimental control and group sizes at the fundamental level, as well as human applicability at a broader translational level. This system is one we have recently established and optimized in our laboratory, and has proven invaluable for gathering yet unpublished and promising data. The reasoning, advantages, and challenges associated with choosing several models are presented below, along with a few practical tips.

\section{From Cells to Tissue}

A classical approach to studying the complexity of the brain is to reduce the complexity to something more easily accessible and controlled, via dissociated neural cell cultures. Neuronal cultures can be generated from a variety of organisms, brain regions, and at different developmental stages, although in biomedical research they are typically produced from mice or rats using perinatal or embryonic neuronal tissue, as young tissue is more resistant to damage and glial cell growth can be more easily controlled. In order to generate neuronal cultures from older animals, for example, specialized dissociation protocols should be used, however the process is more prone to failure ${ }^{94}$. Glial cell cultures, cultures of peripheral neurons, dissociated co-cultures, and cultures from adult animals are also possible. Establishing a typical neuronal culture is done by dissociation, plating, and maintenance of neurons, while they grow in an incubator until maturation, and until weeks after plating, neurons can be seen developing processes and new morphological features. 
Neural cultures have several advantages that make them a good model system to start with, the first of which is the large degree of environmental control compared to the other models. Control over media and growth conditions allows for investigations into the effect of the delivery of controlled concentrations of chosen compounds for tightly controlled periods of time, which can be used to elucidate temporal and concentration based effects of factors and pharmacological agents on neuronal development and function ${ }^{95,96}$. Dissociated cultures also benefit from their relatively easy bright field (figure 6a) and fluorescent observation (figure 6b), due to their low density and nature as a monolayer. For example, in intact tissue, the labelling of cell populations of interest may lead to a large number of densely packed fluorescent neurons, which can hamper morphological analysis and quantification of single cells, making cell culture a valuable alternative ${ }^{97}$. Good microscopic resolution in a monolayer also facilitates subcellular quantification and analyses, such as of specific organelle morphology ${ }^{98-101}$, and protein trafficking ${ }^{102-104}$. Thin monolayer cultures also become more powerful in terms of imaging as imaging techniques themselves become more powerful, and today techniques such as superresolution microscopy ${ }^{105}$ and total internal reflection fluorescence microscopy ${ }^{106}$ are being routinely employed using cell cultures.

a

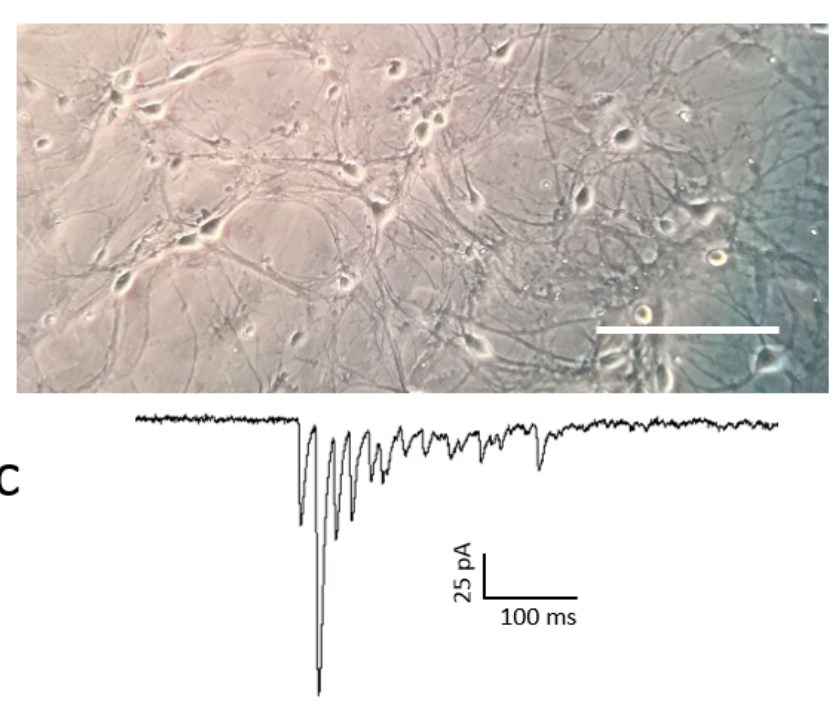

b

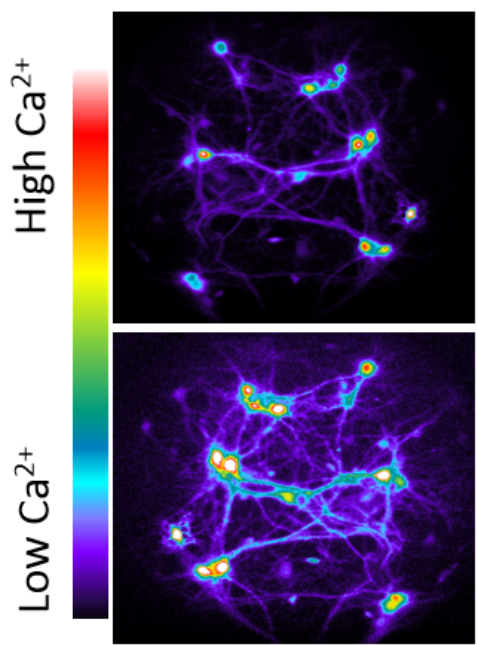

Figure 6. Example of primary mouse cortical neuronal culture. Images show (a) a phase-contrast image of cultured neurons at DIV 14, scale bar=100 $\mu \mathrm{m}$, (b) Fluo-8 fluorescence in a subset of cultured neurons at two time points and (c) Spontaneous synaptic currents measured from one neuron (H. De Castro Abrantes, J.-Y. Chatton, unpublished, Univ. Lausanne).

Electrophysiological studies can also be performed in cell culture (figure $6 \mathrm{c}$ ), which provides several advantages to the experimenter. First, dissociated preparations are simplified models that may be better suited to investigating some mechanisms, since dissociated cells have less uncontrollable 
synaptic inputs and neuromodulatory factors, such as hormones, which are present in intact tissue. Another advantage of cell culture models is their high-throughput capabilities. During a dissection dozens of samples can be generated for upcoming experimentation, and the long-lasting nature of cultures in an incubator means that large data sets can be generated relatively rapidly. Finally, using cell culture also allows cell lysates and extracts to be easily generated from specific cells or cell types for biochemical analyses.

Given the wide range of possible experimental methodologies that can be combined with cell cultures, as well as the large sample sizes, it often makes sense as a first step model system. There are, however, a number of limitations that cannot be overcome without the addition of more complex model systems such as intact tissue. Since a large number of neurological phenomena manifest only at the network level, cells that do not form the necessary synaptic or morphological connections will not recapitulate the phenomenon. While there may still be measurable correlates in the single cell, there may also not, and the emergent property of network interaction will inevitably be lost, leading to questions regarding the relevance of the single-celled measurements. It has also been shown that the degree of network connectivity in a neuronal culture regulates synapse phenotype - changes can be seen in spontaneous release event frequency, kinetics of evoked release, as well as vesicle distribution and release probability ${ }^{24}$. Given that even basic electrophysiological parameters are heavily affected by the culturing process, research that aims to go beyond subcellular neuroscience into cellular and systems neuroscience requires intact tissue.

A good intermediary between dissociated cultures and acute slices in this regard is the organotypic slice. These preparations, like dissociated neurons, can be cultured for extended periods of time. This makes environmental control such as media composition or genetic manipulation easy and approachable. Scalable microfluidic/organotypic slice systems have also been designed for high throughput drug discovery in relation to translational neuroscience ${ }^{107}$, and as a model system they can provide faster data acquisition than the alternative, acute slices. Some control is lost when moving from dissociated cultures to organotypics. Microscopic visualization although possible, is more difficult in thick tissue, which might prevent the visualization of subcellular processes at sufficient resolution in the slice. Organotypics are less difficult than acute slices in this regard, since they flatten out and become thinner over time. Another issue is that not every slice is similar, and since they were each produced by adjacent pieces of tissue without dissociating the cells, some structures will be present in one organotypic slice but not another. Additionally, cell types are necessarily mixed, introducing not only more complexity and difficulty, but also relevance to the in-vivo situation. An example of an experimental difficulty introduced by the necessity for mixed cell types can be seen on the surface of organotypics, where over time a layer of glial cells proliferate to form a thick barrier of cells. 
It is important to highlight some distinctions between organotypics and acute slices that make acute slices additionally valuable. For example, single neuron labelling with fluorescent dyes has revealed a higher degree of neuronal process branching in organotypics compared to acute slices, as well as an increase in the frequency of glutamatergic miniature synaptic currents ${ }^{108}$, an indication that maybe the overall number of synapses is artificially increased in organotypics. Perhaps a result of continued and compensatory development in the face of axotomotical destruction during slicing, this effect may constitute both a drawback and an advantage. As an advantage organotypics may act as a model for research into sprouting and functional recovery after injury ${ }^{109}$. The drawback is reduced homology to the in-vivo situation, which makes acute slices potentially the closest model system to in-vivo conditions when studying events or activity that depend on microcircuitry.

The acute slice is the third model system in our pipeline due to the aforementioned benefits, as it constitutes an ex-situ preparation which is not cultured, and largely represents the in-vivo brain in terms of cytoarchitecture, functioning, and network dynamics, a powerful benefit when aiming for the identification and validation of novel therapeutic agents ${ }^{110}$. Validating findings using acute slices that have been demonstrated with more approachable and high-throughput methods is also a powerful way to increase the impact and potential applicability of the research. The drawbacks of the acute slice are the reason why cell culture and organotypics often precede it- a large number of animals have to be sacrificed to gain statistically significant datasets, every experimental preparation requires a lengthy preparatory phase, and a large degree of experimental control is lost. Although the acute slice, like cell culture, is potentially associated with a large degree of extracellular control, it is not as easy to achieve. Drugs that are applied to the acute slice in the perfusion bath will diffuse slowly through the tissue, and the concentration of any pharmacological compound reached in the center of an acute slice will depend on a number of factors such as diffusion through the slice and slice thickness. Any desired genetic manipulation also has to be performed first in the living animal from which slices were obtained, which is more difficult and time consuming than infection or transfection of an organotypic or cell culture.

\section{From Mouse to Human}

Despite the relative homology between an intact mouse and an acute mouse brain slice, there is always further potential for discordance between rodents and humans. Even though animal models are often hoped or assumed to predict human outcomes and mechanisms, systematic reviews indicate that at a statistical level this predictive power is disputed ${ }^{93,111,112}$. Since animal findings may not constitute predictors, findings that claim to be translational in nature need to be validated in human models. Here we will focus on the acute human brain slice (figure 7), a model we are able to use based on a collaboration with neurosurgeons at a nearby university hospital. While a combination of logistical 
issues has prevented the widespread use of human brain tissue ${ }^{113}$, its use has become increasingly frequent for translational studies, for example in the field of epilepsy research ${ }^{114-117}$.In terms of our model system pipeline, human slices have the additional advantage over IPSCs that they bear some degree of homology to acute rodent slices, and the methodology and equipment used for human slices is very similar or identical, allowing for consistency within a study. Since both forms of slices were resected from living animals and contain original cellular arrangements, the possibility for analogous findings may depend more on the applicability of biological mechanisms to humans rather than to methodological differences. The largest advantage of the human brain slice, however, is that it is the closest representation of the in-vivo human brain outside of a living person, and is one of the only avenues for testing the effect of pharamacological agents or therapies on humans without clinical trials.
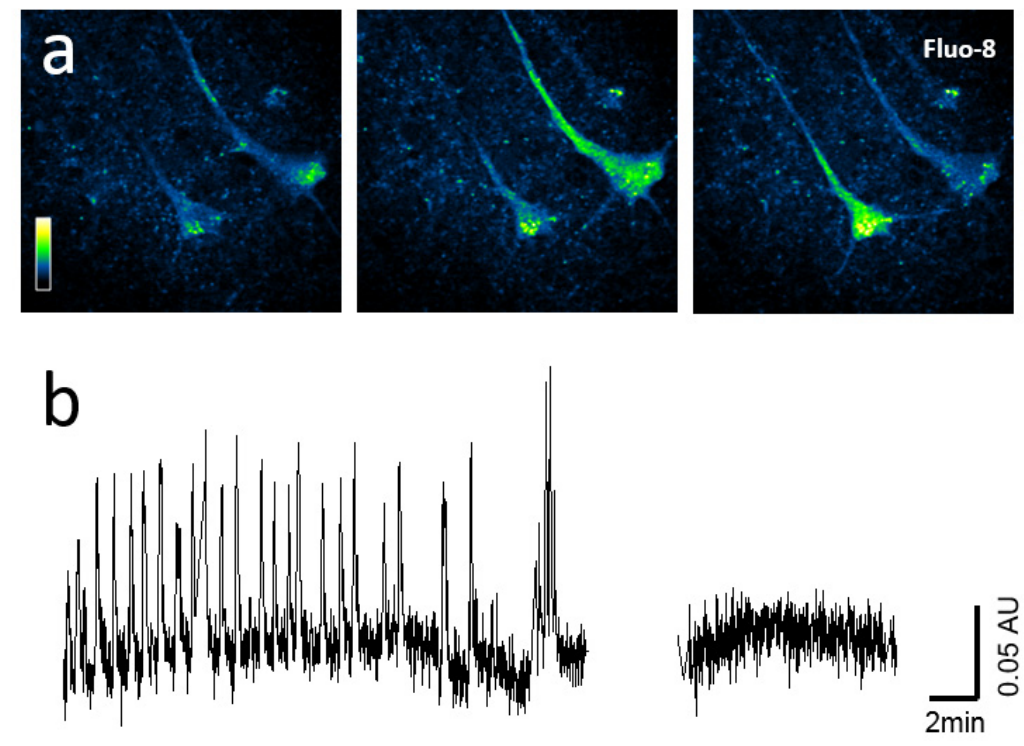

Tetrodotoxin

Figure 7. Human neurons in acute slice. (a)Two-photon images of human neurons loaded with the fluorescent calcium indicator Fluo- 8 in a temporal cortex acute slice $\left(\lambda_{\text {ex }} 810 \mathrm{~nm}\right)$ taken at three different time points. In the false colors images green and yellow correspond to elevated $\mathrm{Ca}^{2+}$. (B) Trace showing the fast fluorescence transients (sampling rate $=1.5 \mathrm{~Hz}$ ). This cell shows spontaneous neuronal activity that was abolished by the addition of the sodium channel blocker tetrodotoxin $(1 \mathrm{uM})$.

While the possibility for insight into human pathology exists with this model system, the degree of experimental control afforded to the researcher is minimal. This is the reason it is the last model system in our pipeline- it provides the most applicability and relevance for translational neuroscience, 
but the least control (it is more useful once a target and experimental methodology have already been established). Access to human tissue is highly restricted and privileged, meaning sample sizes are small, and data takes a long time to gather. The schedule of the research is now subservient to the schedule of the operations. Additionally, patients vary in their age, region of resection, and type as well as degree of pathology. While all human tissue obtained will be to a large extent pathological in nature, this may or may not be desirable depending on the research question and type/degree of pathology. Dead or dying tissue is the occasional reality when dealing with resected human tissue, perhaps in part to the operating procedure or pathology, and perhaps in part to the transportation of the tissue. While the maintenance of tissue can be achieved during short periods of transport in university hospitals tightly linked to research labs, long distance transport may be unfeasible. Additionally, many experimental questions may be unapproachable with this model system, due to the inability to focus on specific brain regions or employ specific methodologies, such as genetic manipulation.

In situations where acute human slices cannot serve as a model system, either due to insufficient infrastructure or experimental demands, IPSC derived human models are a recently publicized alternative, however they lack the multicellular architecture of an acute brain slice, being more akin to normal dissociated cell cultures in terms of advantages and disadvantages. Another altenative and also a recent advancement in human brain slicing is the pathological human organotypic slice ${ }^{118}$. The human organotypic combines the best of human tissue experiments with organotypics, providing an unprecedented degree of control over human tissue in terms of cell-type specific viral infection, longterm imaging, and environmental manipulation, and may serve as a viable alternative to the human brain slice.

\section{Conclusions}

Together the four model systems we presented have allowed us to better develop our mechanistic understanding of both the animal and human brain. Throughout the rich history of neuronal model systems a range of possibilities have emerged, starting with the very first publication of ex-situ mammalian tissue preparations for culturing nerve fibers in 1907. Now, more than a century later, countless models and variations exist, each with their own advantages and disadvantages. Choosing appropriate model systems for neuroscience research is a non-trivial task, in part due to the brains enormous spatial and temporal complexity, as well as our increasingly thorough understanding of its mechanisms and pathologies. Here we hope to have illustrated the need for several model systems, especially in the face of an increasing demand for neurological treatment options, providing an example of a model system pipeline that balances experimental control and human applicability. 


\section{Acknowledgments}

This work was supported by grant \#31003A-179399 from the Swiss National Science Foundation to J.Y. Chatton. We thank Florence Morgenthaler and Haissa de Castro Abrantes for allowing us to modify and use their work.

\section{Author Contributions}

Joel Wellbourne-Wood and Jean-Yves Chatton wrote and edited the manuscript, and produced the figures.

\section{Conflict of Interest}

The authors declare no conflicts of interest. 


\section{References}

1. von Bartheld, C. S., Bahney, J., and Herculano-Houzel, S. (2016) The search for true numbers of neurons and glial cells in the human brain: A review of 150 years of cell counting, J. Comp. Neurol. 524, 3865-3895.

2. Haug, H. (1987) Brain sizes, surfaces, and neuronal sizes of the cortex cerebri: a stereological investigation of man and his variability and a comparison with some mammals (primates, whales, marsupials, insectivores, and one elephant), Am. J. Anat. 180, 126-142.

3. Raichle, M. E., and Gusnard, D. A. (2002) Appraising the brain's energy budget, Proc. Natl. Acad. Sci. USA 99, 10237-10239.

4. Tian, L., and Ma, L. (2017) Microstructural Changes of the Human Brain from Early to Mid-Adulthood, Front. Human Neurosci. 11.

5. Tessier-Lavigne, M., and Goodman, C. S. (1996) The Molecular Biology of Axon Guidance, Science $274,1123-1133$.

6. Schuldiner, O., and Yaron, A. (2015) Mechanisms of developmental neurite pruning, Cell Mol Life Sci 72, 101-119.

7. Harrison, R. (1907) Observations on the living developing nerve fibre., Proc Soc Exp Biol. 4, 140.

8. Carrel, A., and Burrows, M. (1910) Cultivation of adult tissues and organs outside of the body., J. Am.Med. Assoc. 55, 1379-1381.

9. Carrel, A. (1923) A METHOD FOR THE PHYSIOLOGICAL STUDY OF TISSUES IN VITRO, J. Exp. Med. 38, 407-418.

10. Lewis, M., and Lewis, W. (1911) The growth of embryonic chick tissues in artificial media, agar and bouillon, Johns Hopkins Hosp Bull. 22, 116.

11. Ringer, S., and Buxton, D. W. (1887) Upon the Similarity and Dissimilarity of the behaviour of Cardiac and Skeletal Muscle when brought into relation with Solutions containing Sodium, Calcium and Potassium Salts, J. Physiol. (London) 8, 288-295.

12. Eagle, H. (1955) Nutrition needs of mammalian cells in tissue culture, Science 122, 501-514.

13. Freshney, R. I. (2005) Serum-Free Media. In Culture of Animal Cells John Wiley \& Sons, Inc.

14. van der Valk, J., Brunner, D., De Smet, K., Fex Svenningsen, Å., Honegger, P., Knudsen, L. E., Lindl, T., Noraberg, J., Price, A., Scarino, M. L., and Gstraunthaler, G. (2010) Optimization of chemically defined cell culture media - Replacing fetal bovine serum in mammalian in vitro methods, Toxicology in Vitro 24, 1053-1063.

15. Brewer, G. J., Torricelli, J. R., Evege, E. K., and Price, P. J. (1993) Optimized survival of hippocampal neurons in B27-supplemented Neurobasal, a new serum-free medium combination, J. Neurosci. Res. 35, 567-576.

16. Bardy, C., van den Hurk, M., Eames, T., Marchand, C., Hernandez, R. V., Kellogg, M., Gorris, M., Galet, B., Palomares, V., Brown, J., Bang, A. G., Mertens, J., Bohnke, L., Boyer, L., Simon, S., and Gage, F. H. (2015) Neuronal medium that supports basic synaptic functions and activity of human neurons in vitro, Proc. Natl. Acad. Sci. USA 112, E2725-2734.

17. Brewer, G. J., and Price, P. J. (1996) Viable cultured neurons in ambient carbon dioxide and hibernation storage for a month, Neuroreport 7, 1509-1512.

18. Kittler, J. T., Hanley, J. G., and Isaac, J. T. R. (2006) Transfecting and Transducing Neurons with Synthetic Nucleic Acids and Biologically Active Macromolecules. In The Dynamic Synapse: Molecular Methods in lonotropic Receptor Biology (Kittler, J. T., and Moss, S. J., Eds.), Taylor \& Francis Group, LLC., Boca Raton (FL).

19. Doyle, A., McGarry, M. P., Lee, N. A., and Lee, J. J. (2012) The Construction of Transgenic and Gene Knockout/Knockin Mouse Models of Human Disease, Transgenic research 21, 327-349.

20. Furshpan, E. J., MacLeish, P. R., O'Lague, P. H., and Potter, D. D. (1976) Chemical transmission between rat sympathetic neurons and cardiac myocytes developing in microcultures: evidence for cholinergic, adrenergic, and dual-function neurons, Proc. Natl. Acad. Sci. USA 73, 4225-4229.

21. Bourque, M. J., and Trudeau, L. E. (2000) GDNF enhances the synaptic efficacy of dopaminergic neurons in culture, Eur. J. Neurosci. 12, 3172-3180. 
22. Gomperts, S. N., Rao, A., Craig, A. M., Malenka, R. C., and Nicoll, R. A. (1998) Postsynaptically Silent Synapses in Single Neuron Cultures, Neuron 21, 1443-1451.

23. Macis, E., Tedesco, M., Massobrio, P., Raiteri, R., and Martinoia, S. (2007) An automated microdrop delivery system for neuronal network patterning on microelectrode arrays, J. Neurosci. Methods 161, 88-95.

24. Liu, H., Dean, C., Arthur, C. P., Dong, M., and Chapman, E. R. (2009) Autapses and networks of hippocampal neurons exhibit distinct synaptic transmission phenotypes in the absence of synaptotagmin I, J. Neurosci. 29, 7395-7403.

25. Segal, M. M. (1991) Epileptiform activity in microcultures containing one excitatory hippocampal neuron, J. Neurophysiol. 65, 761-770.

26. Morgenthaler, F. D. (2003) Molecular, morphological and functional diversity of single release sites in neurons, In The Faculty of Biology and Medicine, PhD Thesis, University of Lausanne, Lausanne.

27. Morgenthaler, F. D., Knott, G. W., Floyd Sarria, J. C., Wang, X., Staple, J. K., Catsicas, S., and Hirling, $\mathrm{H}$. (2003) Morphological and molecular heterogeneity in release sites of single neurons, Eur. J. Neurosci. 17, 1365-1374.

28. Whitesides, G. M. (2006) The origins and the future of microfluidics, Nature 442, 368-373.

29. Millet, L. J., and Gillette, M. U. (2012) New perspectives on neuronal development via microfluidic environments, Trends Neurosci. 35, 752-761.

30. Siddique, R., and Thakor, N. (2014) Investigation of nerve injury through microfluidic devices, Journal of the Royal Society Interface 11, 20130676.

31. Uzel, S. G. M., Platt, R. J., Subramanian, V., Pearl, T. M., Rowlands, C. J., Chan, V., Boyer, L. A., So, P. T. C., and Kamm, R. D. (2016) Microfluidic device for the formation of optically excitable, threedimensional, compartmentalized motor units, Sci. Adv. 2.

32. Berdichevsky, Y., Staley, K. J., and Yarmush, M. L. (2010) Building and manipulating neural pathways with microfluidics, Lab on a chip 10, 999-1004.

33. Kunze, A., Meissner, R., Brando, S., and Renaud, P. (2011) Co-pathological connected primary neurons in a microfluidic device for Alzheimer studies, Biotech. Bioeng. 108, 2241-2245.

34. Virlogeux, A., Moutaux, E., Christaller, W., Genoux, A., Bruyère, J., Fino, E., Charlot, B., Cazorla, M., and Saudou, F. (2018) Reconstituting Corticostriatal Network on-a-Chip Reveals the Contribution of the Presynaptic Compartment to Huntington's Disease, Cell Rep. 22, 110-122.

35. Houlgatte, R., Mallat, M., Brachet, P., and Prochiantz, A. (1989) Secretion of nerve growth factor in cultures of glial cells and neurons derived from different regions of the mouse brain, J. Neurosci. Res. 24, 143-152.

36. Ullian, E. M., Harris, B. T., Wu, A., Chan, J. R., and Barres, B. A. (2004) Schwann cells and astrocytes induce synapse formation by spinal motor neurons in culture, Mol. Cell. Neurosci. 25, 241-251.

37. Cao, G., and Ko, C.-P. (2007) Schwann Cell-Derived Factors Modulate Synaptic Activities at Developing Neuromuscular Synapses, J. Neurosci. 27, 6712-6722.

38. Le Roux, P. D., and Reh, T. A. (1994) Regional differences in glial-derived factors that promote dendritic outgrowth from mouse cortical neurons in vitro, The Journal of neuroscience : the official journal of the Society for Neuroscience 14, 4639-4655.

39. Le Berre-Scoul, C., Chevalier, J., Oleynikova, E., Cossais, F., Talon, S., Neunlist, M., and Boudin, H. (2017) A novel enteric neuron-glia coculture system reveals the role of glia in neuronal development, J. Physiol. (London) 595, 583-598.

40. Elmariah, S. B., Oh, E. J., Hughes, E. G., and Balice-Gordon, R. J. (2005) Astrocytes regulate inhibitory synapse formation via Trk-mediated modulation of postsynaptic GABAA receptors, J. Neurosci. 25, 3638-3650.

41. Jones, E. V., Cook, D., and Murai, K. K. (2012) A neuron-astrocyte co-culture system to investigate astrocyte-secreted factors in mouse neuronal development, Meth, Mol. Biol. 814, 341-352.

42. Skaper, S. D., and Facci, L. (2012) Central Nervous System Neuron-Glia Co-culture Models. In Neurotrophic Factors: Methods and Protocols (Skaper, S. D., Ed.) pp 79-89, Humana Press, Totowa, NJ. 
43. Kawano, H., Katsurabayashi, S., Kakazu, Y., Yamashita, Y., Kubo, N., Kubo, M., Okuda, H., Takasaki, K., Kubota, K., Mishima, K., Fujiwara, M., Harata, N. C., and Iwasaki, K. (2012) Long-Term Culture of Astrocytes Attenuates the Readily Releasable Pool of Synaptic Vesicles, PloS ONE 7, e48034.

44. Ricoult, S. G., Goldman, J. S., Stellwagen, D., Juncker, D., and Kennedy, T. E. (2012) Generation of microisland cultures using microcontact printing to pattern protein substrates, J. Neurosci. Methods 208, 10-17.

45. Shi, M., Majumdar, D., Gao, Y., Brewer, B., Goodwin, C. R., McLean, J. A., Li, D., and Webb, D. J. (2013) Glia Co-Culture with Neurons in Microfluidic Platforms Promotes the Formation and Stabilization of Synaptic Contacts, Lab on a chip 13, 3008-3021.

46. Majumdar, D., Gao, Y., Li, D., and Webb, D. J. (2011) Co-Culture of Neurons and Glia in a Novel Microfluidic Platform, J. Neurosci. Methods 196, 38-44.

47. Efremova, L., Schildknecht, S., Adam, M., Pape, R., Gutbier, S., Hanf, B., Bürkle, A., and Leist, M. (2015) Prevention of the degeneration of human dopaminergic neurons in an astrocyte co-culture system allowing endogenous drug metabolism, Br. J. Pharmacol. 172, 4119-4132.

48. Appelt-Menzel, A., Cubukova, A., Günther, K., Edenhofer, F., Piontek, J., Krause, G., Stüber, T., Walles, H., Neuhaus, W., and Metzger, M. (2017) Establishment of a Human Blood-Brain Barrier Coculture Model Mimicking the Neurovascular Unit Using Induced Pluri- and Multipotent Stem Cells, Stem Cell Rep. 8, 894-906.

49. Takahashi, K., and Yamanaka, S. (2006) Induction of Pluripotent Stem Cells from Mouse Embryonic and Adult Fibroblast Cultures by Defined Factors, Cell 126, 663-676.

50. Takahashi, K., Tanabe, K., Ohnuki, M., Narita, M., Ichisaka, T., Tomoda, K., and Yamanaka, S. (2007) Induction of pluripotent stem cells from adult human fibroblasts by defined factors, Cell 131, 861872.

51. Yu, J., Vodyanik, M. A., Smuga-Otto, K., Antosiewicz-Bourget, J., Frane, J. L., Tian, S., Nie, J., Jonsdottir, G. A., Ruotti, V., Stewart, R., Slukvin, I. I., and Thomson, J. A. (2007) Induced Pluripotent Stem Cell Lines Derived from Human Somatic Cells, Science 318, 1917-1920.

52. Hou, S., and Lu, P. (2016) Direct reprogramming of somatic cells into neural stem cells or neurons for neurological disorders, Neur. Regen. Res. 11, 28-31.

53. Gascon, S., Masserdotti, G., Russo, G. L., and Gotz, M. (2017) Direct Neuronal Reprogramming: Achievements, Hurdles, and New Roads to Success, Cell stem cell 21, 18-34.

54. Zhang, S. Z., Ma, L. X., Qian, W. J., Li, H. F., Wang, Z. F., Wang, H. X., and Wu, Z. Y. (2016) Modeling Neurological Disease by Rapid Conversion of Human Urine Cells into Functional Neurons, Stem Cells Int. 2016, 2452985.

55. Mc, I. H., Buchel, L., and Cheshire, J. D. (1951) The inorganic phosphate and phosphocreatine of Brain especially during metabolism in vitro, Biochem. J. 48, 12-20.

56. Yamamoto, C., and Mcllwain, H. (1966) Electrical activities in thin sections from the mammalian brain maintained in chemically-defined media in vitro, J. Neurochem. 13, 1333-1343.

57. Collingridge, G. L. (1995) The brain slice preparation: a tribute to the pioneer Henry Mcllwain, J. Neurosci. Methods 59, 5-9.

58. Huang, S., and Uusisaari, M. (2013) Physiological temperature during brain slicing enhances the quality of acute slice preparations, Front. Cell. Neurosci. 7.

59. Ting, J. T., Daigle, T. L., Chen, Q., and Feng, G. (2014) Acute brain slice methods for adult and aging animals: application of targeted patch clampanalysis and optogenetics, Meth, Mol. Biol. 1183, 221242.

60. Bosch, D., Asede, D., and Ehrlich, I. (2016) Ex Vivo Optogenetic Dissection of Fear Circuits in Brain Slices, J. Vis. Exp., e53628.

61. Agmon, A., and Connors, B. W. (1991) Thalamocortical responses of mouse somatosensory (barrel) cortex in vitro, Neuroscience 41, 365-379.

62. Itami, C., Huang, J.-Y., Yamasaki, M., Watanabe, M., Lu, H.-C., and Kimura, F. (2016) Developmental Switch in Spike Timing-Dependent Plasticity and Cannabinoid-Dependent Reorganization of the Thalamocortical Projection in the Barrel Cortex, The Journal of Neuroscience 36, 7039-7054. 
63. Arnson, H. A., and Strowbridge, B. W. (2017) Spatial Structure of Synchronized Inhibition in the Olfactory Bulb, J. Neurosci. 37, 10468-10480.

64. Pouille, F., McTavish, T. S., Hunter, L. E., Restrepo, D., and Schoppa, N. E. (2017) Intraglomerular gap junctions enhance interglomerular synchrony in a sparsely connected olfactory bulb network, J. Physiol. (London) 595, 5965-5986.

65. Strauch, C., and Manahan-Vaughan, D. (2018) In the Piriform Cortex, the Primary Impetus for Information Encoding through Synaptic Plasticity Is Provided by Descending Rather than Ascending Olfactory Inputs, Cereb. Cortex 28, 764-776.

66. Maier, N., Güldenagel, M., Söhl, G., Siegmund, H., Willecke, K., and Draguhn, A. (2002) Reduction of high-frequency network oscillations (ripples) and pathological network discharges in hippocampal slices from connexin 36-deficient mice, J. Physiol. (London) 541, 521-528.

67. Pálhalmi, J., Paulsen, O., Freund, T. F., and Hájos, N. (2004) Distinct properties of carbachol- and DHPG-induced network oscillations in hippocampal slices, Neuropharmacol. 47, 381-389.

68. Sheridan, G. K., Moeendarbary, E., Pickering, M., O'Connor, J. J., and Murphy, K. J. (2014) ThetaBurst Stimulation of Hippocampal Slices Induces Network-Level Calcium Oscillations and Activates Analogous Gene Transcription to Spatial Learning, PloS ONE 9, e100546.

69. Rimmele, T. S., Rocher, A. B., Wellbourne-Wood, J., and Chatton, J. Y. (2017) Control of Glutamate Transport by Extracellular Potassium: Basis for a Negative Feedback on Synaptic Transmission, Cereb. Cortex, 1-12.

70. Buskila, Y., Breen, P. P., Tapson, J., van Schaik, A., Barton, M., and Morley, J. W. (2014) Extending the viability of acute brain slices, Sci Rep 4, 5309.

71. Kelava, I., and Lancaster, M. A. (2016) Dishing out mini-brains: Current progress and future prospects in brain organoid research, Dev. Biol. 420, 199-209.

72. Lee, C.-T., Bendriem, R. M., Wu, W. W., and Shen, R.-F. (2017) 3D brain Organoids derived from pluripotent stem cells: promising experimental models for brain development and neurodegenerative disorders, J. Biomed. Sci. 24, 59.

73. Stoppini, L., Buchs, P. A., and Muller, D. (1991) A simple method for organotypic cultures of nervous tissue, J Neurosci Methods 37, 173-182.

74. Wolf, M. K. (1970) Anatomy of cultured mouse cerebellum. II. Organotypic migration of granule cells demonstrated by silver impregnantion of normal and mutant cultures, J. Comp. Neurol. 140, 281-298.

75. Giesing, M., Neumann, G., Egge, H., and Zilliken, F. (1975) Lipid metabolism of developing central nervous tissues in organotypic cultures. I. Lipid distribution and fatty acid profiles of the medium for rat brain cortex in vitro, Nutr. Metab. 19, 242-250.

76. Whetsell, W. O., Jr., Mytilineou, C., Shen, J., and Yahr, M. D. (1981) The development of the dog nigrostriatal system in organotypic culture, J. Neural. Transm. 52, 149-161.

77. Ostergaard, K., Finsen, B., and Zimmer, J. (1995) Organotypic slice cultures of the rat striatum: an immunocytochemical, histochemical and in situ hybridization study of somatostatin, neuropeptide Y, nicotinamide adenine dinucleotide phosphate-diaphorase, and enkephalin, Exp. Brain. Res. 103, 70-84.

78. Michel, V., Monnier, Z., Cvetkovic, V., and Math, F. (1999) Organotypic culture of neuroepithelium attached to olfactory bulb from adult mouse as a tool to study neuronal regeneration after $\mathrm{ZnSO} 4$ neuroepithelial trauma, Neurosci. Lett. 271, 195-198.

79. Gahwiler, B. H. (1981) Morphological differentiation of nerve cells in thin organotypic cultures derived from rat hippocampus and cerebellum, Proc R Soc Lond B Biol Sci 211, 287-290.

80. Chong, Y. H., Shin, Y. J., Lee, E. O., Kayed, R., Glabe, C. G., and Tenner, A. J. (2006) ERK1/2 activation mediates Abeta oligomer-induced neurotoxicity via caspase-3 activation and tau cleavage in rat organotypic hippocampal slice cultures, J. Biol. Chem. 281, 20315-20325.

81. Nassif, M., Hoppe, J., Santin, K., Frozza, R., Zamin, L. L., Simao, F., Horn, A. P., and Salbego, C. (2007) Beta-amyloid peptide toxicity in organotypic hippocampal slice culture involves Akt/PKB, GSK3beta, and PTEN, Neurochem. Int. 50, 229-235. 
82. Bruce, A. J., Malfroy, B., and Baudry, M. (1996) beta-Amyloid toxicity in organotypic hippocampal cultures: protection by EUK-8, a synthetic catalytic free radical scavenger, Proc. Natl. Acad. Sci. USA 93, 2312-2316.

83. Johansson, S., Jamsa, A., Vasange, M., Winblad, B., Luthman, J., and Cowburn, R. F. (2006) Increased tau phosphorylation at the Ser396 epitope after amyloid beta-exposure in organotypic cultures, Neuroreport 17, 907-911.

84. Marksteiner, J., and Humpel, C. (2008) Beta-amyloid expression, release and extracellular deposition in aged rat brain slices, Mol Psychiatry 13, 939-952.

85. Humpel, C., and Weis, C. (2002) Nerve growth factor and cholinergic CNS neurons studied in organotypic brain slices. Implication in Alzheimer's disease?, Journal of neural transmission. Supplementum, 253-263.

86. Cavaliere, F., Vicente, E. S., and Matute, C. (2010) An organotypic culture model to study nigrostriatal degeneration, J. Neurosci. Methods 188, 205-212.

87. Heine, C., and Franke, H. (2014) Organotypic slice co-culture systems to study axon regeneration in the dopaminergic system ex vivo, Meth, Mol. Biol. 1162, 97-111.

88. Daviaud, N., Garbayo, E., Lautram, N., Franconi, F., Lemaire, L., Perez-Pinzon, M., and MonteroMenei, C. N. (2014) MODELING NIGROSTRIATAL DEGENERATION IN ORGANOTYPIC CULTURES, A NEW EX VIVO MODEL OF PARKINSON'S DISEASE, Neuroscience 256, 10-22.

89. Brana, C., Benham, C. D., and Sundstrom, L. E. (1999) Calpain activation and inhibition in organotypic rat hippocampal slice cultures deprived of oxygen and glucose, Eur. J. Neurosci. 11, 2375-2384.

90. Pringle, A. K., lannotti, F., Wilde, G. J. C., Chad, J. E., Seeley, P. J., and Sundstrom, L. E. (1997) Neuroprotection by both NMDA and non-NMDA receptor antagonists in in vitro ischemia, Brain Res. 755, 36-46.

91. Morin-Brureau, M., De Bock, F., and Lerner-Natoli, M. (2013) Organotypic brain slices: a model to study the neurovascular unit micro-environment in epilepsies, Fluids and Barriers of the CNS 10,1111.

92. Falsig, J., Sonati, T., Herrmann, U. S., Saban, D., Li, B., Arroyo, K., Ballmer, B., Liberski, P. P., and Aguzzi, A. (2012) Prion Pathogenesis Is Faithfully Reproduced in Cerebellar Organotypic Slice Cultures, PLoS Pathogens 8, e1002985.

93. Mak, I. W. Y., Evaniew, N., and Ghert, M. (2014) Lost in translation: animal models and clinical trials in cancer treatment, Am. J. Translat.Res. 6, 114-118.

94. Brewer, G. J., and Torricelli, J. R. (2007) Isolation and culture of adult neurons and neurospheres, Nat. Protoc. 2, 1490-1498.

95. Burkhalter, J., Fiumelli, H., Allaman, I., Chatton, J.-Y., and Martin, J.-L. (2003) Brain-Derived Neurotrophic Factor Stimulates Energy Metabolism in Developing Cortical Neurons, J. Neurosci. 23, 8212-8220.

96. Gang, L., Yao, Y.-C., Liu, Y.-f., Li, Y.-p., Yang, K., Lu, L., Cheng, Y.-c., Chen, X.-y., and Tu, Y. (2015) Coculture of oligodendrocytes and neurons can be used to assess drugs for axon regeneration in the central nervous system, Neur. Regen. Res. 10, 1612-1616.

97. Pleiser, S., Banchaabouchi, M. A., Samol-Wolf, A., Farley, D., Welz, T., Wellbourne-Wood, J., Gehring, I., Linkner, J., Faix, J., Riemenschneider, M. J., Dietrich, S., and Kerkhoff, E. (2014) Enhanced fear expression in Spir-1 actin organizer mutant mice, Eur. J. Cell Biol. 93, 225-237.

98. Rao, A., Simmons, D., and Sorkin, A. (2011) Differential subcellular distribution of endosomal compartments and the dopamine transporter in dopaminergic neurons, Mol. Cell. Neurosci. 46, 148-158.

99. Wiemerslage, L., and Lee, D. (2016) Quantification of Mitochondrial Morphology in Neurites of Dopaminergic Neurons using Multiple Parameters, J. Neurosci. Methods 262, 56-65.

100. Thayer, D. A., Jan, Y. N., and Jan, L. Y. (2013) Increased neuronal activity fragments the Golgi complex, PNAS 110, 1482-1487.

101. Stepanova, T., Slemmer, J., Hoogenraad, C. C., Lansbergen, G., Dortland, B., De Zeeuw, C. I., Grosveld, F., van Cappellen, G., Akhmanova, A., and Galjart, N. (2003) Visualization of microtubule 
growth in cultured neurons via the use of EB3-GFP (end-binding protein 3-green fluorescent protein), The Journal of Neuroscience 23, 2655-2664.

102. Al-Bassam, S., Xu, M., Wandless, T. J., and Arnold, D. B. (2012) Differential trafficking of transport vesicles contributes to the localization of dendritic proteins, Cell Rep. 2, 89-100.

103. Lipka, J., Kapitein, L. C., Jaworski, J., and Hoogenraad, C. C. (2016) Microtubule-binding protein doublecortin-like kinase 1 (DCLK1) guides kinesin-3-mediated cargo transport to dendrites, The EMBO Journal 35, 302-318.

104. Bisbal, M., Conde, C., Donoso, M., Bollati, F., Sesma, J., Quiroga, S., Añel, A. D., Malhotra, V., Marzolo, M. P., and Cáceres, A. (2008) Protein Kinase D Regulates Trafficking of Dendritic Membrane Proteins in Developing Neurons, The Journal of Neuroscience 28, 9297-9308.

105. Huang, B., Bates, M., and Zhuang, X. (2009) Super resolution fluorescence microscopy, Annu. Rev. Biochem. 78, 993-1016.

106. Mattheyses, A. L., Simon, S. M., and Rappoport, J. Z. (2010) Imaging with total internal reflection fluorescence microscopy for the cell biologist, J. Cell Sci. 123, 3621-3628.

107. Jing, L., Liping, P., Cheng, X., and Berdichevsky, Y. (2016) Perfused drop microfluidic device for brain slice culture-based drug discovery, Biomed. Microdev. 18, 46-46.

108. De Simoni, A., Griesinger, C. B., and Edwards, F. A. (2003) Development of rat CA1 neurones in acute Versus organotypic slices: role of experience in synaptic morphology and activity, J. Physiol. (London) 550, 135-147.

109. Stoppini, L., Parisi, L., Oropesa, C., and Muller, D. (1997) Sprouting and functional recovery in cocultures between old and young hippocampal organotypic slices, Neuroscience 80, 1127-1136.

110. Cho, S., Wood, A., and Bowlby, M. R. (2007) Brain Slices as Models for Neurodegenerative Disease and Screening Platforms to Identify Novel Therapeutics, Curr. Neuropharmacol. 5, 19-33.

111. Shanks, N., Greek, R., and Greek, J. (2009) Are animal models predictive for humans?, Philosophy, Ethics, and Humanities in Medicine: PEHM 4, 2-2.

112. Knight, A. (2007) Systematic reviews of animal experiments demonstrate poor human clinical and toxicological utility, Altern Lab Anim 35, 641-659.

113. Jones, R. S., da Silva, A. B., Whittaker, R. G., Woodhall, G. L., and Cunningham, M. O. (2016) Human brain slices for epilepsy research: Pitfalls, solutions and future challenges, J. Neurosci. Methods 260, 221-232.

114. Klaft, Z. J., Hollnagel, J. O., Salar, S., Calişkan, G., Schulz, S. B., Schneider, U. C., Horn, P., Koch, A., Holtkamp, M., Gabriel, S., Gerevich, Z., and Heinemann, U. (2016) Adenosine A1 receptor-mediated suppression of carbamazepine-resistant seizure-like events in human neocortical slices, Epilepsia $57,746-756$.

115. Huberfeld, G., Menendez de la Prida, L., Pallud, J., Cohen, I., Le Van Quyen, M., Adam, C., Clemenceau, S., Baulac, M., and Miles, R. (2011) Glutamatergic pre-ictal discharges emerge at the transition to seizure in human epilepsy, Nat. Neurosci. 14, 627.

116. Gabriel, S., Njunting, M., Pomper, J. K., Merschhemke, M., Sanabria, E. R. G., Eilers, A., Kivi, A., Zeller, M., Meencke, H.-J., Cavalheiro, E. A., Heinemann, U., and Lehmann, T.-N. (2004) Stimulus and Potassium-Induced Epileptiform Activity in the Human Dentate Gyrus from Patients with and without Hippocampal Sclerosis, The Journal of Neuroscience 24, 10416-10430.

117. Bedner, P., Dupper, A., Huttmann, K., Muller, J., Herde, M. K., Dublin, P., Deshpande, T., Schramm, J., Haussler, U., Haas, C. A., Henneberger, C., Theis, M., and Steinhauser, C. (2015) Astrocyte uncoupling as a cause of human temporal lobe epilepsy, Brain 138, 1208-1222.

118. Le Duigou, C., Savary, E., Morin-Brureau, M., Gomez-Dominguez, D., Sobczyk, A., Chali, F., Milior, G., Kraus, L., Meier, J. C., Kullmann, D. M., Mathon, B., de la Prida, L. M., Dorfmuller, G., Pallud, J., Eugene, E., Clemenceau, S., and Miles, R. (2018) Imaging pathological activities of human brain tissue in organotypic culture, J. Neurosci. Methods 298, 33-44. 